\title{
Offline estimation of decay time for an optical cavity with a low pass filter cavity model
}

\author{
Abhijit G. Kallapur, * Toby K. Boyson, Ian R. Petersen, and Charles C. Harb \\ School of Engineering and IT, UNSW@ADFA, Northcott Drive, Canberra, ACT, Australia \\ ${ }^{*}$ Corresponding author: abhijit.kallapur@gmail.com
}

Received April 24, 2012; revised June 12, 2012; accepted June 13, 2012;

posted June 13, 2012 (Doc. ID 166563); published July 16, 2012

\begin{abstract}
This Letter presents offline estimation results for the decay-time constant for an experimental Fabry-Perot optical cavity for cavity ring-down spectroscopy (CRDS). The cavity dynamics are modeled in terms of a low pass filter (LPF) with unity DC gain. This model is used by an extended Kalman filter (EKF) along with the recorded light intensity at the output of the cavity in order to estimate the decay-time constant. The estimation results using the LPF cavity model are compared to those obtained using the quadrature model for the cavity presented in previous work by Kallapur et al. The estimation process derived using the LPF model comprises two states as opposed to three states in the quadrature model. When considering the EKF, this means propagating two states and a $(2 \times 2)$ covariance matrix using the LPF model, as opposed to propagating three states and a $(3 \times 3)$ covariance matrix using the quadrature model. This gives the former model a computational advantage over the latter and leads to faster execution times for the corresponding EKF. It is shown in this Letter that the LPF model for the cavity with two filter states is computationally more efficient, converges faster, and is hence a more suitable method than the three-state quadrature model presented in previous work for real-time estimation of the decay-time constant for the cavity. (C) 2012 Optical Society of America

OCIS codes: $300.6360,050.2230,200.3050$.
\end{abstract}

The Fabry-Perot optical cavity used in this Letter consists of a stainless steel spacer, separating two highly reflecting mirrors. Tunable laser light $(1550 \mathrm{~nm})$ is coupled into the cavity, then terminated at regular intervals. The measured output field has a ring-up and ringdown profile, which is a characteristic of the cavity parameters and losses between the mirrors, and the spectroscopic technique is termed cavity ring-down spectroscopy (CRDS); e.g., see [1-4]. In this Letter, a continuous-wave (CW) laser source is used and the optical cavity is actively locked to the frequency of the incident light [2]. The time it takes for the light intensity inside the cavity to decay to $1 / e$ times its initial intensity is termed as the decay time and is denoted by $\tau$. This rate of decay of light intensity inside the cavity depends on the reflectivity of the mirrors inside the cavity and losses due to sample(s) contained within the cavity that directly dictate the amount of absorption. If the absorption due to the mirrors is measured in advance for a vacuum cavity, the absorption due to the sample within the cavity can be computed explicitly. The rate of decay of the light as a function of wavelength may be used to generate a spectrum, and may thus be used for detecting various molecules and compounds and as a detector for chromatographic systems.

Linear methods such as the conventional linear least squares and nonlinear least square methods such as the Levenberg-Marquardt (LM) can be used to estimate the value for $\tau$. However, the linear methods can be used to estimate $\tau$ only in the case of isolated ring-downs and are susceptible to instrument offsets and system noise characteristics [5-8] Also, the LM method is known to limit data throughput to below $10 \mathrm{~Hz}$ [7-9]. An offline estimation scheme for $\tau$ is presented in $[\overline{7}, \underline{8}]$, where the cavity is modeled in terms of the annihilation and creation operators reformulated in terms of the amplitude and phase quadratures. An extended Kalman filter (EKF) was then applied to the measured intensity data at the output of the cavity for the estimation of $\tau$. Though this method provides a good estimate for $\tau$, it requires the input signal to be DC coupled and is applicable only to locked experiments. In this Letter, we model the cavity as a low pass filter (LPF), which is computationally less expensive than the model used in $[7,8]$, can handle both $\mathrm{AC}$ as well as DC coupled inputs, and is applicable to both $\mathrm{CW}$ as well as pulsed inputs. We also compare the estimation results for this method with the estimation scheme used in $[\underline{7}, \underline{8}]$. A detailed description of the experimental setup used in this Letter along with an introduction to the application of modern estimation and control techniques can be found in $[7,8]$.

Traditional CRDS data processing techniques fit experimental data to an exponential equation described by

$$
I(t)=I_{0} \exp (-t / \tau)
$$

where $I(t)$ represents the decay intensity at time $t, I_{0}$ is the initial light intensity at the output of the cavity, and $\tau$ is the decay time. Many curve fitting techniques apply Eq. (1) in order to compute the value for $\tau$. However, most of these techniques are not suitable for real-time estimation of $\tau$; see, e.g., [5-9]. It was shown in $[7,8]$ that modern estimation techniques such as the EKF can be used to estimate $\tau$ in real-time. In order to apply the theory of the EKF, the dynamics of the optical cavity is modeled in terms of the annihilation and creation operators as $[\underline{7}, \underline{8}, \underline{10}, \underline{11}]$ :

$$
\dot{a}=-\left(\frac{\gamma}{2}+i \Delta\right) a-\sqrt{\gamma_{m}}\left(\bar{a}_{\text {in }}+w\right) ; \quad y=\gamma_{m} a^{\dagger} a+v .
$$


Here, $a$ denotes the annihilation operator for the cavity mode defined in an appropriate rotating frame, $(\cdot)^{\dagger}$ represents the operator adjoint operation, and $\gamma=\gamma_{m}+$ $\gamma_{c}$ is the total cavity coupling coefficient. $\gamma_{m}$ represents the cavity coupling coefficient at the mirrors in a vacuum cavity and $\gamma_{c}$ represents the cavity coupling coefficient corresponding to the absorbers within the cavity. Also, $\Delta$ is the detuning parameter, $\bar{a}_{\text {in }}$ is the laser input, $y$ is the measured output corresponding to the output light intensity, and $w$ and $v$ represent lumped process and measurement noise terms, respectively.

As shown in $[\underline{7}, \underline{8}]$, the cavity dynamics in Eq. (2) can be reformulated in terms of quadrature variables for amplitude and phase. The resultant equations are written in the state space form before applying the EKF for estimating $\tau$. In this Letter, we model the dynamics of the optical cavity as an LPF. This model is motivated by the fact that, with respect to intensity modulation on the input light, the cavity operates like an LPF; e.g., see $[\underline{12}, \underline{13}]$. The cavity LPF model is represented in the form of the transfer function,

$$
G(s)=\gamma /(s+\gamma),
$$

where $\gamma=1 / \tau$ is the coupling coefficient. This can be written in the continuous-time state space form as

$$
\begin{aligned}
& \dot{x}(t)=-\gamma x(t)+u(t)+w(t), \\
& y(t)=\gamma x(t)+v(t),
\end{aligned}
$$

where $x(t)$ is the state of the system, $u(t)$ is the input (laser), and $y(t)$ is the output. Also, $w(t)$ and $v(t)$ are the lumped process and measurement noise terms, respectively, that represent the noise components in the input signal, from measurement devices, and other noise sources. Since we are interested in estimating the value of $\tau=1 / \gamma$, we can consider $\gamma$ to be a state of the system and augment it with the original state $x(t)$ in Eq. (4). Setting $x_{1}(t)=x(t)$ and $x_{2}(t)=\gamma$, the system (4) and ( $\left.\underline{5}\right)$ can be written in the nonlinear form

$$
\left[\begin{array}{l}
\dot{x}_{1}(t) \\
\dot{x}_{2}(t)
\end{array}\right]=\left[\begin{array}{cc}
-x_{2}(t) & 0 \\
0 & 0
\end{array}\right]\left[\begin{array}{l}
x_{1}(t) \\
x_{2}(t)
\end{array}\right]+\left[\begin{array}{l}
1 \\
0
\end{array}\right] u(t)+\left[\begin{array}{l}
w_{1}(t) \\
w_{2}(t)
\end{array}\right],
$$

$$
y(t)=x_{1}(t) x_{2}(t)+v(t) .
$$

Here, in the absence of noise, $\dot{x}_{2}=0$ because $\gamma$ is a constant. This system is in the general nonlinear form

$$
\begin{aligned}
& \dot{x}(t)=f_{c}(x(t), u(t))+D_{c} w(t), \\
& y(t)=h(x(t))+v(t) .
\end{aligned}
$$

Since the measurement is obtained in discrete time steps, we can write the dynamics in the discrete time form as $[\underline{7}, \underline{8}]$

$$
x_{k}=f\left(x_{k}, u_{k}\right)+D_{d} w_{k},
$$

$$
y_{k+1}=h\left(x_{k+1}\right)+v_{k+1} .
$$

Also, the discrete time measurement equation is computed as

$$
y_{k+1}=h\left(x_{k+1}\right)+v_{k+1}=x_{1_{k+1}} x_{2_{k+1}}+v_{k+1} .
$$
by

\section{Propagation}

$$
\begin{aligned}
& x_{k+1}^{-}=f\left(x_{k}^{+}, u_{k}\right), \\
& P_{k+1}^{-}=F_{k} P_{k}^{+} F_{k}^{T}+Q \delta .
\end{aligned}
$$

Update

$$
\begin{aligned}
K_{k+1} & =P_{k+1}^{-} H_{k+1}^{T}\left(H_{k+1} P_{k+1}^{-} H_{k+1}^{T}+R\right)^{-1}, \\
x_{k+1}^{+} & =x_{k+1}^{-}+K_{k+1}\left(y_{k+1}-h\left(x_{k+1}^{-}\right)\right), \\
P_{k+1}^{+} & =I-K_{k+1} H_{k+1} P_{k+1}^{-} .
\end{aligned}
$$

Here, the propagation step consists of estimating the value of the state $x$ and error covariance $P$ (a matrix representing the approximate variance of the estimate of the state from its true value) one time-step ahead. These values are computed using available state(s) and input(s) at the current time-step and evaluating the state dynamics $f\left(x_{k}^{+}, u_{k}\right)$. The errors in propagation are then corrected using the measured sensor value(s) in the update step. Also, in Eqs. (13)-(17), $y_{(\cdot)}$ is the measured sensor output and $F_{(\cdot)}, H_{(\cdot)}$ are the linearized process and output matrices, respectively, computed about the current operating point as

$$
F_{k}=\left.\frac{\partial f(x, u)}{\partial x}\right|_{x=x_{k}^{+}} ; \quad H_{k+1}=\left.\frac{\partial h(x)}{\partial x}\right|_{x=x_{k+1}^{-}} .
$$

Indeed, since $f(x, u)$ and $h(x)$ are smooth differentiable functions, the linearization in Eq. (18) is well defined. In addition, $K(\cdot)$ represents the Kalman gain, $P$ is the error covariance matrix, $Q$ and $R$ are the process and measurement noise covariance matrices, and $I$ is the identity matrix of suitable dimensions. Also, $(\cdot)^{-}$and $(\cdot)^{+}$represent $a$ priori and a posteriori values, respectively, and $\delta$ is the sampling time constant.

The actual estimation process was carried out by applying the discrete time EKF recursion equations (13)(17) to the experimentally obtained light intensity data at the output of the cavity. Various matrices and constants in Eqs. (13)-(17) were chosen as part of the design process and were set to be

$$
\begin{aligned}
& Q=\left[\begin{array}{cc}
9.98 \times 10^{-17} & 0 \\
0 & 0
\end{array}\right] ; R=10^{-8} \\
& P_{0}=\left[\begin{array}{cc}
9.98 \times 10^{-17} & 0 \\
0 & 10^{5}
\end{array}\right] ; x_{0}=\left[\begin{array}{c}
0 \\
1.9 \times 10^{4}
\end{array}\right] .
\end{aligned}
$$


Here, $P_{0}$ is the initial error covariance matrix and $x_{0}$ is the initial state vector. In addition, the system in Eqs. (6) and (7) was linearized with respect to the state vector $\left[x_{1}(t), x_{2}(t)\right]^{T}$ as

$$
\begin{aligned}
& \frac{\partial f(x, u)}{\partial x}=\left[\begin{array}{cc}
-x_{2}(t) & -x_{1}(t) \\
0 & 0
\end{array}\right], \\
& \frac{\partial h(x)}{\partial x}=\left[x_{2}(t) \quad x_{1}(t)\right] .
\end{aligned}
$$

The system in Eq. (20) was discretized using the Euler scheme described in $[\underline{7}, \underline{8}]$ and evaluated at $x=x_{k}^{+}$. During the estimation process, the initial guess for $\gamma$ was set at $10 \%$ of its expected true value corresponding to $90 \%$ error in initial condition. The measured light intensity at the output of the cavity was stored in the vector $y(\cdot)$ defined in Eq. (12), and the sampling time constant $\delta$ was set to $10^{-8} \mathrm{~s}$. For a detailed discussion on the definition of matrices $Q, P$, and $R$ along with their physical significance, see, e.g., [14].

A detailed description of the experimental setup used to record light intensity data at the output of the FabryPerot cavity can be found in $[7,8]$. During the process of estimation for $\tau$, the laser light coupling to the input of the cavity was chopped at $25 \mathrm{kHz}$, and the corresponding light intensity at the output was recorded and applied as measurements to the discrete time EKF recursion equations (13)-(17) using constants outlined in Eq. (19). The estimation process was carried out using a thousand run Monte Carlo (MC) procedure, programmed using Matlab. At each MC run, the EKF was applied to the same set of measured intensity outputs but with different sets of randomly generated input noise values. A sample EKF estimation output for $\tau$ using the method described in this Letter is compared with the EKF estimation results presented in $[\underline{7}, \underline{8}]$.

From Fig. 1, though both methods provide acceptable estimation results, the EKF estimation for $\tau$, where the cavity is modeled as in $[7,8]$, takes longer to settle close to the expected value of $\approx 5.3 \mu \mathrm{s}$. On the other hand, the EKF estimation for $\tau$ using an LPF model for the cavity dynamics settles close to the expected value faster. In addition, since the LPF model for the cavity consists of two states in the augmented state vector, as compared to three states in the model presented in $[7,8]$, in the filter (EKF) formulation with the LPF cavity model, only two states need to be propagated along with a $(2 \times 2)$ covariance matrix with each filter recursion. This is computationally less expensive than propagating three states and a $(3 \times 3)$ covariance matrix as in the case of the quadrature model in [7,8] [see Eqs. (13) and (14)]. This makes the estimation process using the LPF cavity model more suitable for real-time implementation. A comparison of the average execution times for both methods is outlined in Table 1 .

In conclusion, though the $\tau$ estimation process presented in $[7,8]$ and the LPF approach presented in this Letter provide acceptable results, it is seen that the latter

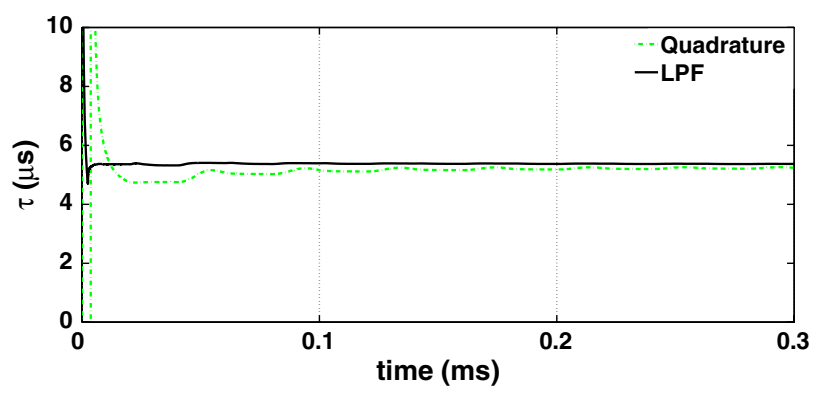

Fig. 1. (Color online) Comparison of EKF estimation result for $\tau$ : quadrature model in $[\underline{7,}, \underline{8}]$ versus LPF model for the cavity.

Table 1. Comparison of Average EKF Execution Times From 1000 MC Runs

\begin{tabular}{lcc}
\hline & Quadrature model & LPF model \\
\hline Total time & $1.65 \mathrm{~s}$ & $1.5 \mathrm{~s}$ \\
One recursion & $41.3 \mu \mathrm{s}$ & $37.5 \mu \mathrm{s}$ \\
\hline
\end{tabular}

uses a simpler model for cavity dynamics, has a faster EKF execution time, settles close to the expected value for $\tau$ within fewer ring-down cycles, and exhibits smaller overshoot in the beginning of the estimation process than the former method.

\section{References}

1. A. O'Keefe and D. A. G. Deacon, Rev. Sci. Instrum. 59, 2544 (1988).

2. B. A. Paldus, C. C. Harb, T. G. Spence, B. Wilke, J. Xie, J. S. Harris, and R. N. Zare, J. Appl. Phys. 83, 3991 (1998).

3. K. W. Busch and M. A. Busch, Cavity-Ringdown Spectroscopy. An Ultratrace-Absorption Measurement Technique, vol. 720 of ACS Symposium Series (American Chemical Society, 1999).

4. T. G. Spence, C. C. Harb, B. A. Paldus, R. N. Zare, B. Willke, and R. L. Byer, Rev. Sci. Instrum. 71, 347 (2000).

5. A. A. Istratov and O. F. Vyvenko, Rev. Sci. Instrum. 70, 1233 (1999).

6. M. Mazurenka, R. Wada, A. J. L. Shillings, T. J. A. Butler, J. M. Beames, and A. J. Orr-Ewing, Appl. Phys. B: Lasers Opt. 81, 135 (2005).

7. A. G. Kallapur, T. K. Boyson, I. R. Petersen, and C. C. Harb, in IEEE International Conference on Control Applications (CCA) (IEEE, 2011), p. 556.

8. A. G. Kallapur, T. K. Boyson, I. R. Petersen, and C. C. Harb, Opt. Express 19, 6377 (2011).

9. M. A. Everest and D. B. Atkinson, Rev. Sci. Instrum. 79, 023108 (2008).

10. C. W. Gardiner and P. Zoller, Quantum Noise (Springer, 2000).

11. H. A. Bachor and T. C. Ralph, A Guide to Experiments in Quantum Optics, 2nd ed. (Wiley-VCH, 2004).

12. J. M. Herbelin, J. A. McKay, M. A. Kwok, R. H. Ueunten, D. S. Urevig, D. J. Spencer, and D. J. Benard, Appl. Opt. 19, 144 (1980).

13. R. Engeln, G. V. Helden, G. Berden, and G. Meijer, Chem. Phys. Lett. 262, 105 (1996).

14. Y. Bar-Shalom and X. Li, Estimation and Tracking: Principles, Techniques, and Software (Artech House, 1993). 